\title{
On best proximity points of upper semicontinuous multivalued mappings
}

\author{
Ghada AINemer ${ }^{1,2}$, Jack Markin ${ }^{3}$ and Naseer Shahzad ${ }^{4 *}$
}

"Correspondence:

nshahzad@kau.edu.sa

${ }^{4}$ Operator Theory and Applications

Research Group, Department of

Mathematics, Faculty of Science, King Abdulaziz University, P.O. Box

80203, Jeddah, 21589, Saudi Arabia

Full list of author information is

available at the end of the article

\begin{abstract}
In this paper we study the existence of best proximity points of a nonself upper semicontinuous multivalued mapping $T: A \rightarrow 2^{B}$ in a strictly convex Banach space. This multivalued mapping commutes with affine, noncyclic, and relatively $u$-continuous single-valued mapping $f: A \cup B \rightarrow A \cup B$. Also, we study the case when $T$ commutes with a family of commuting, affine, noncyclic, and relatively $u$-continuous single-valued mappings on $A \cup B$. Moreover, we present some examples to illustrate our results.
\end{abstract}

MSC: $47 \mathrm{H} 10 ; 54 \mathrm{H} 25$

Keywords: best proximity point; multivalued mapping; fixed point; upper semicontinuous mapping; relatively u-continuous mapping

\section{Introduction}

Let $A, B$ be nonempty subsets of a metric space $(X, d)$ and $T: A \rightarrow 2^{B}$, where $2^{B}$ is the family of all nonempty subsets of $B$. If $A \cap B=\emptyset$, the operator inclusion $x \in T(x)$ has no solution. In this case, it is logical to look for a point $x \in A$ such that $\operatorname{dist}(x, T(x))$ is minimum. Because $\operatorname{dist}(x, T(x))$ is at least $\operatorname{dist}(A, B)$, the point $x$ is the solution of the equation $\operatorname{dist}(x, T(x))=\operatorname{dist}(A, B)=\inf \{d(x, y): x \in A, y \in B\}$. This point is called the best proximity point of $T$. Indeed, best proximity point theorems examine the existence of such optimal approximate solutions of the operator inclusion $x \in T(x)$ when there is no exact solution. If $A \cap B \neq \emptyset$, the best proximity point is the fixed point of $T$.

For multivalued mappings, the existence of best proximity points was established by many authors, e.g., Abkar and Gabeleh in [1] and [2], Al-Thagafi and Shahzad in [3], Amini-Harandi in [4], De la Sen in [5], Kirk et al. in [6] and Włodarczyk et al. in [7]. Best proximity point theorems for relatively nonexpansive single-valued mapping were studied in [8] in 2005. Since then there has been a lot of activity in this area and a number of results appeared by various authors. Best proximity point theorems for relatively $u$-continuous mapping were proved in [9] and [10]. For other related results, we refer the reader to [11-16] and [17]. In this paper, we study the existence of best proximity points for an upper semicontinuous multivalued mapping with nonempty, compact, and convex values $T: A \rightarrow 2^{B}$ which commutes with an affine and relatively $u$-continuous single-valued mapping $f: A \cup B: \rightarrow A \cup B$ such that $f(A) \subseteq A$ and $f(B) \subseteq B$ (noncyclic). In addition, we present some support examples for our results and we also give an example showing

(c) 2015 AlNemer et al. This article is distributed under the terms of the Creative Commons Attribution 4.0 International License (http://creativecommons.org/licenses/by/4.0/), which permits unrestricted use, distribution, and reproduction in any medium, provided you give appropriate credit to the original author(s) and the source, provide a link to the Creative Commons license, and indicate if changes were made. 
that the condition ' $T(x) \cap B_{0} \neq \emptyset$ for each $x \in A_{0}$ ' is necessary. Moreover, we add a similar theorem for a multivalued mapping which commutes with a family of commuting, affine, noncyclic, and relatively $u$-continuous single-valued mappings on $A \cup B$.

\section{Preliminaries}

Definition 2.1 [9] Let $A, B$ be nonempty subsets of a metric space $X$. A mapping $f: A \cup$ $B \rightarrow A \cup B$ is said to be relatively $u$-continuous if for each $\epsilon>0$ there exists $\delta>0$ such that $d(f(x), f(y))<\epsilon+\operatorname{dist}(A, B)$ whenever $d(x, y)<\delta+\operatorname{dist}(A, B)$ for each $x \in A, y \in B$.

Definition 2.2 [8] Let $A, B$ be nonempty subsets of a metric space $X$. A mapping $f: A \cup$ $B \rightarrow A \cup B$ is called relatively nonexpansive if $d(f(x), f(y)) \leq d(x, y)$ for each $x \in A, y \in B$.

Every relatively nonexpansive mapping is relatively $u$-continuous. However, the converse is not true (see [9]).

Definition 2.3 [3] Let $A, B$ be nonempty subsets of a metric space $X$ and $T: A \rightarrow 2^{B}$ a multivalued mapping. A point $x \in A$ is called a (i) fixed point of $T$ if $x \in T(x)$ and (ii) best proximity point of $T$ if $\operatorname{dist}(x, T(x))=\operatorname{dist}(A, B)$. Note that if $\operatorname{dist}(A, B)=0$, then we get a fixed point of $T$.

Definition 2.4 Let $A, B$ be nonempty subsets of a metric space $X$. A multivalued mapping $T: A \rightarrow 2^{B}$ is called upper semicontinuous if $T^{-1}(C)=\{x \in A: T(x) \cap C \neq \emptyset\}$ is closed in $A$ whenever $C$ is closed in $B$.

Proposition 2.5 [18] Let $X$ be a strictly convex Banach space, A a nonempty, compact, and convex subset of $X$, and $B$ a nonempty closed subset of $X$. Let $\left\{x_{n}\right\}$ be a sequence in $A$ and $y \in B$. If $\left\|x_{n}-y\right\| \rightarrow \operatorname{dist}(A, B)$, then $x_{n} \rightarrow P_{A}(y)$.

Definition 2.6 [9] Let $A, B$ be nonempty convex subsets of a Banach space $X$. A mapping $f: A \cup B \rightarrow A \cup B$ is called affine if $f(\alpha x+\beta y)=\alpha f(x)+\beta f(y)$ for all $x, y \in A$ or $x, y \in B$ and $\alpha, \beta \in[0,1]$ with $\alpha+\beta=1$.

Lemma 2.7 [19] If $A$ is a nonempty, compact, and convex subset of a Banach space, and $T: A \rightarrow 2^{A}$ can be expressed as a composition of finitely many upper semicontinuous multivalued mappings with nonempty, compact, and convex values, then $T$ has a fixed point.

Let $A, B$ be nonempty subsets of a Banach space $X . f: A \cup B \rightarrow A \cup B$ a relatively nonexpansive mapping such that $f(A) \subseteq A, f(B) \subseteq B, T: A \rightarrow \mathrm{KC}(B)$, where $\mathrm{KC}(B)$ is the set of all nonempty, compact, and convex subsets of $B$. The mapping $f$ and $T$ are said to commute if for each $x \in A, f(T(x)) \subseteq T(f(x))$. Define

$$
\begin{aligned}
& A_{0}=\{x \in A:\|x-y\|=\operatorname{dist}(A, B) \text { for some } y \in B\}, \\
& B_{0}=\{y \in B:\|x-y\|=\operatorname{dist}(A, B) \text { for some } x \in A\} .
\end{aligned}
$$

Remark 2.8 Note that if $A$ and $B$ are nonempty, compact, and convex sets, then $A_{0}$ and $B_{0}$ are nonempty, compact, and convex sets with $\operatorname{dist}\left(A_{0}, B_{0}\right)=\operatorname{dist}(A, B)$. For details see [6] and [8]. Also, $f\left(A_{0}\right) \subseteq A_{0}$ and $f\left(B_{0}\right) \subseteq B_{0}[10]$. 
Remark 2.9 [3,9] Let $A$ be a nonempty subset of a normed space $X$. The metric projection operator is defined by $P_{A}(x)=\{y \in A:\|x-y\|=\operatorname{dist}(x, A)\}$ for each $x \in X$. If $A$ is a nonempty, compact, and convex subset of a Banach space $X$, then $P_{A}$ is upper semicontinuous with nonempty, compact, and convex values. Observe that when $A$ is a nonempty, compact, and convex subset of a strictly convex Banach space $X, P_{A}$ is a single-valued mapping from $X$ to $A$.

Theorem 2.10 [10] Let $A, B$ be nonempty, compact, and convex subsets of a strictly convex Banach space $X$. If $: A \cup B: \rightarrow A \cup B$ is relatively $u$-continuous such that $f(A) \subseteq A$ and $f(B) \subseteq B$. Then there exists $\left(x_{0}, y_{0}\right) \in A \times B$ such that $f\left(x_{0}\right)=x_{0}, f\left(y_{0}\right)=y_{0}$, and $\left\|x_{0}-y_{0}\right\|=$ $\operatorname{dist}(A, B)$.

\section{Main results}

The following proposition is a noncyclic version of Proposition 3.2 in [9].

Proposition 3.1 Let $A, B$ be nonempty, compact, and convex subsets of a strictly convex Banach space $X$. Let $f: A \cup B \rightarrow A \cup B$ be a relatively u-continuous mapping such that $f(A) \subseteq A$ and $f(B) \subseteq B . P: A \cup B \rightarrow A \cup B$ is a mapping defined by

$$
P(x)= \begin{cases}P_{B}(x) & \text { if } x \in A, \\ P_{A}(x) & \text { if } x \in B .\end{cases}
$$

Then $f(P(x))=P(f(x))$ for each $x \in A_{0} \cup B_{0}$, i.e., $P_{A}(f(y))=f\left(P_{A}(y)\right)$ for each $y \in B_{0}$ and $P_{B}(f(x))=f\left(P_{B}(x)\right)$ for each $x \in A_{0}$.

Proof Let $x \in A_{0}$. Then there exists $y \in B$ such that $\|x-y\|=\operatorname{dist}(A, B)$. So, $y=P_{B}(x)$ and $x=P_{A}(y)$. Then for each $\delta>0,\|x-y\|<\delta+\operatorname{dist}(A, B)$. Since $f$ is relatively $u$-continuous, for each $\epsilon>0$ we have $\operatorname{dist}(A, B) \leq\|f(x)-f(y)\|<\epsilon+\operatorname{dist}(A, B)$. Thus, $\|f(x)-f(y)\|=$ $\operatorname{dist}(A, B)$. So, $f(x)=P_{A}(f(y))$ and $f(y)=P_{B}(f(x))$. Since $A, B$ are nonempty, compact, and convex subsets of a strictly convex Banach space, the metric projection is unique. Now, $x=P_{A}(y) \Longrightarrow f(x)=f\left(P_{A}(y)\right) \Longrightarrow P_{A}(f(y))=f\left(P_{A}(y)\right)$ for each $y \in B_{0}$. Also, $y=P_{B}(x) \Longrightarrow$ $f(y)=f\left(P_{B}(x)\right) \Longrightarrow P_{B}(f(x))=f\left(P_{B}(x)\right)$ for each $x \in A_{0}$. Hence, $f(P(x))=P(f(x))$ for each $x \in A_{0} \cup B_{0}$.

A cyclic version of the following proposition can be found in [9] (see the proof of Theorem 3.1 in [9]).

Proposition 3.2 Let $A, B$ be nonempty, compact, and convex subsets of a strictly convex Banach space $X$. Let $f: A \cup B \rightarrow A \cup B$ be a relatively u-continuous mapping such that $f(A) \subseteq A$ and $f(B) \subseteq B$. Then $f$ is continuous on $A_{0}$ and $B_{0}$.

Proof Let $x_{0} \in A_{0}$ and $\left\{x_{n}\right\} \subseteq A_{0}$ such that $x_{n} \rightarrow x_{0}$. We want to show that $f\left(x_{n}\right) \rightarrow f\left(x_{0}\right)$. Using the triangle inequality, we obtain

$$
\begin{aligned}
\left\|x_{n}-P_{B}\left(x_{0}\right)\right\| & \leq\left\|x_{n}-x_{0}\right\|+\left\|x_{0}-P_{B}\left(x_{0}\right)\right\| \\
& =\left\|x_{n}-x_{0}\right\|+\operatorname{dist}(A, B) \\
& \rightarrow \operatorname{dist}(A, B) .
\end{aligned}
$$


Then for each $\delta>0$ there exists $N_{0} \in \mathbb{N}$ such that for each $n \geq N_{0}$, we have \|\|$x_{n}-P_{B}\left(x_{0}\right) \|-$ $\operatorname{dist}(A, B) \mid<\delta$. So, $n \geq N_{0} \Longrightarrow\left\|x_{n}-P_{B}\left(x_{0}\right)\right\|<\delta+\operatorname{dist}(A, B)$. By relative $u$-continuity of $f$, $\left\|f\left(x_{n}\right)-f\left(P_{B}\left(x_{0}\right)\right)\right\|<\epsilon+\operatorname{dist}(A, B)$ for each $n \geq N_{0}$. Since $\left\{f\left(x_{n}\right)\right\} \subseteq A$ and $P_{B}\left(f\left(x_{0}\right)\right) \in B$, Proposition 2.5 gives

$$
f\left(x_{n}\right) \rightarrow P_{A}\left(f\left(P_{B}\left(x_{0}\right)\right)\right)=f\left(P_{A}\left(P_{B}\left(x_{0}\right)\right)\right)=f\left(x_{0}\right)
$$

Hence, $f\left(x_{n}\right) \rightarrow f\left(x_{0}\right)$. Since $x_{0} \in A_{0}$ was arbitrary, $f$ is continuous on $A_{0}$. Similarly, $f$ is continuous on $B_{0}$. Therefore, $f$ is continuous on $A_{0} \cup B_{0}$.

Theorem 3.3 Let $A, B$ be nonempty, compact, and convex subsets in a strictly convex Banach space $X$. Suppose $f: A \cup B \rightarrow A \cup B$ is an affine relatively u-continuous mapping with $f(A) \subseteq A, f(B) \subseteq B$. Then there exists $\left(x_{0}, y_{0}\right) \in A \times B$ such that $f\left(x_{0}\right)=x_{0}, f\left(y_{0}\right)=y_{0}$ and $\left\|x_{0}-y_{0}\right\|=\operatorname{dist}(A, B)$.

In addition, if $T: A \rightarrow \mathrm{KC}(B)$ is an upper semicontinuous multivalued mapping, $f$ and $T$ commute, and $T(x) \cap B_{0} \neq \emptyset$ for each $x \in A_{0}$, then there exists $a \in A$ such that $f(a)=a$ and $\operatorname{dist}(a, T(a))=\operatorname{dist}(A, B)$.

Proof For $u \in A_{0}$, there is a $v \in B$ such that $\|u-v\|=\operatorname{dist}(A, B)$. Then by the relative $u$-continuity of $f,\|f(u)-f(v)\|=\operatorname{dist}(A, B)$, implying that $f(u) \in A_{0}$. Therefore, the compact convex set $A_{0}$ is invariant under the continuous mapping $f$, and the Schauder fixed point theorem implies the existence of a fixed point $x_{0}=f\left(x_{0}\right) \in A_{0}$. Let $y_{0}$ be the unique closest point to $x_{0}$ in $B$. Then by the relative $u$-continuity of $f$ and the uniqueness of the closest point projection onto $B, y_{0}=f\left(y_{0}\right)$ and $\left\|x_{0}-y_{0}\right\|=\operatorname{dist}(A, B)$.

Now, we will prove that there exists $a \in A$ such that $\operatorname{dist}(a, T(a))=\operatorname{dist}(A, B)$. Define $\operatorname{Fix}(f)=\{x \in A \cup B: f(x)=x\}, \operatorname{Fix}_{A}(f)=\operatorname{Fix}(f) \cap A_{0}$ and $\operatorname{Fix}_{B}(f)=\operatorname{Fix}(f) \cap B_{0}$. Clearly, $\operatorname{Fix}_{A}(f)$ and $\operatorname{Fix}_{B}(f)$ are nonempty, because $x_{0} \in \operatorname{Fix}_{A}(f)$ and $y_{0} \in \operatorname{Fix}_{B}(f)$. The set $\operatorname{Fix}_{A}(f)$ is closed. Indeed, let $\left\{x_{n}\right\} \subseteq \operatorname{Fix}_{A}(f)$ such that $x_{n} \rightarrow x_{0}$. Since $\left\{x_{n}\right\} \subseteq A_{0}$ and $A_{0}$ is closed by Remark 2.8, we have $x_{0} \in A_{0} \subseteq A$. Using Proposition 3.2, $f\left(x_{n}\right) \rightarrow f\left(x_{0}\right)$. But $f\left(x_{n}\right)=x_{n}$ for each $n$. So $x_{n} \rightarrow f\left(x_{0}\right)$. Consequently $x_{0}=f\left(x_{0}\right)$. Thus $x_{0} \in \operatorname{Fix}_{A}(f)$. Therefore, $\operatorname{Fix}_{A}(f)$ is closed. Similarly, $\operatorname{Fix}_{B}(f)$ is closed. So, $\operatorname{Fix}_{A}(f)$ and $\operatorname{Fix}_{B}(f)$ are compact sets as they are closed subsets of the compact sets $A_{0}, B_{0}$. In addition, $\operatorname{Fix}_{A}(f)$ is a convex set. Indeed, let $x, y \in \operatorname{Fix}_{A}(f)$ and $\alpha, \beta \in[0,1]$ with $\alpha+\beta=1$. Since $f$ is affine, $f(\alpha x+\beta y)=\alpha f(x)+$ $\beta f(y)=\alpha x+\beta y$, i.e., $\alpha x+\beta y \in \operatorname{Fix}(f)$. Also, $\alpha x+\beta y \in A_{0}$ as $A_{0}$ is convex and $x, y \in A_{0}$. Consequently, $\alpha x+\beta y \in \operatorname{Fix}(f) \cap A_{0}=\operatorname{Fix}_{A}(f)$. Similarly, $\operatorname{Fix}_{B}(f)$ is a convex set.

Assume $x \in \operatorname{Fix}_{A}(f)$ and choose $v \in T(x)$. Since $f$ and $T$ commute, $f(v) \in T(f(x))=T(x)$, which implies that $T(x)$ is invariant under $f$. Then the invariance of $B_{0}$ under $f$ shows that the compact convex set $T(x) \cap B_{0}$ is invariant under $f$. Since $f$ is continuous on $B_{0}$, by the Schauder fixed point theorem $f$ has a fixed point in $T(x) \cap B_{0}$, implying that $T(x) \cap$ $\operatorname{Fix}_{B}(f) \neq \emptyset$ for each $x \in \operatorname{Fix}_{A}(f)$.

Now, define $F: \operatorname{Fix}_{A}(f) \rightarrow 2^{\operatorname{Fix}_{B}(f)}$ by $F(x)=T(x) \cap \operatorname{Fix}_{B}(f)$ for each $x \in \operatorname{Fix}_{A}(f)$. Then $F$ is an upper semicontinuous multivalued mapping with nonempty, compact, and convex values. Note that $P_{A}: \operatorname{Fix}_{B}(f) \rightarrow \operatorname{Fix}_{A}(f)$. To see this, let $x \in \operatorname{Fix}_{B}(f) \subseteq B_{0}$. Then there exists $y \in A$ such that $\|x-y\|=\operatorname{dist}(A, B)$. So, $y=P_{A}(x)$ and $x=P_{B}(y)$. For each $\delta>0$, we have $\|x-y\|<\delta+\operatorname{dist}(A, B)$. Using the relative $u$-continuity for any $f, \operatorname{dist}(A, B) \leq\|f(x)-f(y)\|<$ $\epsilon+\operatorname{dist}(A, B)$ for each $\epsilon>0$. Thus, $\|f(x)-f(y)\|=\operatorname{dist}(A, B)$. This implies that $f(y)=P_{A}(f(x))$ 
and $f(x)=P_{B}(f(y))$. Since $x \in \operatorname{Fix}_{B}(f)$ and $y=P_{A}(x)$, we have $f(y)=f\left(P_{A}(x)\right)=P_{A}(f(x))=$ $P_{A}(x)$ and so $P_{A}(x) \in \operatorname{Fix}_{A}(f) \subseteq A$. Note that $P_{A} \circ F: \operatorname{Fix}_{A}(f) \rightarrow 2^{\mathrm{Fix}_{A}(f)}$. By Lemma 2.7, there exists $a \in \operatorname{Fix}_{A}(f) \subseteq A_{0}$ such that $a \in\left(P_{A} \circ F\right)(a)$, i.e., $a=f(a)$ and $a \in P_{A}(F(a))$. So, there exists $b \in F(a)=T(a) \cap \operatorname{Fix}_{B}(f) \subseteq B_{0}$ such that $a=P_{A}(b) \subseteq \operatorname{Fix}_{A}(f)$. As $a=P_{A}(b)$, $\|a-b\|=\operatorname{dist}(b, A)$. Since $b \in F(a)=T(a) \cap \operatorname{Fix}_{B}(f) \subseteq B_{0}$, then $b \in T(a)$ and $b \in B_{0}$. Since $b \in B_{0}$, there exists $a^{\prime} \in A$ such that $\left\|a^{\prime}-b\right\|=\operatorname{dist}(A, B)$. Since $a \in A$ and $T(a) \subseteq B$, we have

$$
\begin{aligned}
\operatorname{dist}(A, B) & \leq \operatorname{dist}(a, T(a)) \\
& \leq\|a-b\| \\
& =\operatorname{dist}(b, A) \\
& \leq\left\|b-a^{\prime}\right\| \\
& =\operatorname{dist}(A, B) .
\end{aligned}
$$

Thus, $\operatorname{dist}(a, T(a))=\operatorname{dist}(A, B)$.

Remark 3.4 The condition $T(x) \cap B_{0} \neq \emptyset$ for each $x \in A_{0}$ is necessary in Theorem 3.3. For example, in the real space if $A=[1,5] \times[-5,5], B=\left[-1, \frac{-1}{25}\right] \times[-5,5]$. Define

$$
f: A \cup B \rightarrow A \cup B \text { by } f(x, y)=\left(x, \frac{y+1}{2}\right)
$$

and

$$
T: A \rightarrow \mathrm{KC}(B) \quad \text { by } \quad T(x, y)=\left[-1, \frac{-1}{x^{2}}\right] \times\{y\} .
$$

Clearly, $T$ is upper semicontinuous and $f$ is affine and relatively $u$-continuous. Also, $f(A) \subseteq A$ and $f(B) \subseteq B$. There are fixed points of $f, x_{0}=(1,1) \in A, y_{0}=\left(\frac{-1}{25}, 1\right) \in B$ such that $\left\|x_{0}-y_{0}\right\|=\operatorname{dist}(A, B)=1.04$. In addition, $f$ and $T$ commute. Suppose that there exists $a \in \operatorname{Fix}(f) \cap A$ such that $\operatorname{dist}(a, T(a))=1.04$. Then $a=(z, 1)$, for some $1 \leq z \leq 5$. So,

$$
\operatorname{dist}(a, T(a))=\operatorname{dist}\left((z, 1),\left[-1, \frac{-1}{z^{2}}\right] \times\{1\}\right)=\left\|(z, 1)-\left(\frac{-1}{z^{2}}, 1\right)\right\|=1.04 .
$$

Consequently, $z^{3}-1.04 z^{2}+1=0$. So, $z_{1}=0.893939214944+0.7334769205376 i, z_{2}=$ $0.893939214944-0.7334769205376 i$, which are not real numbers, and $z_{3}=$ -0.747878429888 , which does not belong to $[1,5]$. Note that $A_{0}=\{1\} \times[-5,5], B_{0}=\left\{\frac{-1}{25}\right\} \times$ $[-5,5]$. For $x=(1, y) \in A_{0}$, we have $T(x)=T(1, y)=\{(-1, y)\}$. So, $T(x) \cap B_{0}=\{(-1, y)\} \cap$ $\left\{\left(\frac{-1}{25}, y\right):-5 \leq y \leq 5\right\}=\emptyset$.

Corollary 3.5 Let A, B be nonempty, compact, and convex sets in a strictly convex Banach space $X$. If $T: A \rightarrow \mathrm{KC}(B)$ is an upper semicontinuous multivalued mapping and $T(x) \cap$ $B_{0} \neq \emptyset$ for each $x \in A_{0}$, then there exists $a \in A$ such that $\operatorname{dist}(a, T(a))=\operatorname{dist}(A, B)$.

Proof Taking $f=I$ (the identity mapping on $A \cup B$ ) in Theorem 3.3, we obtain the desired result. 
Corollary 3.6 Let A be a nonempty, compact, and convex set in a strictly convex Banach space. Suppose $f: A \rightarrow A$ is an affine continuous mapping. If $T: A \rightarrow \mathrm{KC}(A)$ is an upper semicontinuous multivalued mapping and $f, T$ commute, then there exists $a \in A$ such that $a \in \operatorname{Fix}(f) \cap \operatorname{Fix}(T)$.

Proof Since any continuous mapping on a compact set is relatively $u$-continuous on that set, taking $A=B$ in Theorem 3.3, we see that there exists $a \in \mathrm{A}$ such that $f(a)=a$ and $\operatorname{dist}(a, T(a))=\operatorname{dist}(A, A)=0$, i.e., $a \in T(a)$. So, $f(a)=a \in T(a)$. Therefore, $a \in \operatorname{Fix}(f) \cap$ $\operatorname{Fix}(T)$.

Theorem 3.7 Let $X$ be a strictly convex Banach space. Let A, B be nonempty, compact, and convex subsets of $X$ and let $f, g: A \cup B \rightarrow A \cup B$ be commuting, affine, and relatively $u$-continuous mappings such that $f(A) \subseteq A, f(B) \subseteq B$ and $g(A) \subseteq A, g(B) \subseteq B$. Then there exist points $x_{0} \in A$ and $y_{0} \in B$ such that $x_{0}=f\left(x_{0}\right)=g\left(x_{0}\right), y_{0}=f\left(y_{0}\right)=g\left(y_{0}\right)$ and $\left\|x_{0}-y_{0}\right\|=$ $\operatorname{dist}(A, B)$.

Proof For $u \in A_{0}$, there is a $v \in B$ such that $\|u-v\|=\operatorname{dist}(A, B)$. Then by the relative $u$-continuity of $f,\|f(u)-f(v)\|=\operatorname{dist}(A, B)$, implying that $f(u) \in A_{0}$. Therefore, the compact convex set $A_{0}$ is invariant under the continuous mapping $f$, and the Schauder fixed point theorem implies the existence of a fixed point $x=f(x) \in A_{0}$. The set of fixed points of $f$ in $A_{0}$ (denoted by $\operatorname{Fix}_{A}(f)$ ) is closed and convex since $f$ is continuous and affine. If $x \in \operatorname{Fix}_{A}(f)$, commutativity of $f$ and $g$ implies $f(g(x))=g(f(x))=g(x)$. Therefore, Fix $_{A}(f)$ is invariant under $g$, and since $g$ is continuous it has a fixed point in $\operatorname{Fix}_{A}(f)$. Let $x_{0}$ be a common fixed point of $f$ and $g$ in $A_{0}$, that is, $x_{0}=f\left(x_{0}\right)=g\left(x_{0}\right)$, and let $y_{0}$ be the unique closest point to $x_{0}$ in $B$. Then by the relative $u$-continuity of $f$ and $g$ and the uniqueness of the closest point projection onto $B, y_{0}=f\left(y_{0}\right)=g\left(y_{0}\right)$ and $\left\|x_{0}-y_{0}\right\|=\operatorname{dist}(A, B)$.

The previous theorem can be extended to an arbitrary family of commuting affine and noncyclic mappings. The proof depends on the following common fixed point result for commuting affine $u$-continuous mappings in strictly convex Banach spaces. The proof of this result is adapted from Przebieracz ([20], Theorem 1.1) and is included for convenience of the reader.

Lemma 3.8 (Markov-Kakutani theorem) Let $X$ be a strictly convex Banach space. Let A, $B$ be nonempty, compact, and convex subsets of $X$ and let $\mathfrak{F}$ be a family of commuting affine and relatively u-continuous mappings on $A \cup B$ such that $f(A) \subseteq A$ and $f(B) \subseteq B$. Then there is an $x_{0} \in A_{0}$ such that $f\left(x_{0}\right)=x_{0}$ for every $f \in \mathfrak{F}$. There is a $y_{0} \in B_{0}$ such that $f\left(y_{0}\right)=y_{0}$ for every $f \in \mathfrak{F}$.

Proof Notice that the mappings in the family $\mathfrak{F}$ are continuous on $A_{0} \cup B_{0}$. Let $\operatorname{Fix}(f)=$ $\{x \in A \cup B: f(x)=x\}, \operatorname{Fix}_{A}(f)=\operatorname{Fix}(f) \cap A_{0}, f \in \mathfrak{F}$. As shown in the proof of Theorem 3.7, $\operatorname{Fix}_{A}(f) \neq \emptyset$ and $\operatorname{Fix}_{A}(f)$ is convex and compact. To prove that $\bigcap_{f \in \mathfrak{F}} \operatorname{Fix}_{A}(f) \neq \emptyset$, consider any finite collection from $\mathfrak{F}$, say $f_{1}, \ldots, f_{n}$. Assume that

$$
C=\bigcap_{1 \leq i \leq n} \operatorname{Fix}_{A}\left(f_{i}\right) \neq \emptyset .
$$


For each $x \in C$ and $k \in\{1, \ldots, n\}, f_{k} f_{n+1}(x)=f_{n+1} f_{k}(x)=f_{n+1}(x)$, which implies that $f_{n+1}(x) \in C$. Therefore, the compact convex set $C$ is invariant under $f_{n+1}$, implying that $\operatorname{Fix}_{A}\left(f_{n+1}\right) \cap C \neq$ $\emptyset$ since $f_{n+1}$ is continuous on $A_{0}$. Since every finite collection of the sets $\operatorname{Fix}_{A}(f), f \in \mathfrak{F}$, has a nonempty intersection, we have $\bigcap_{f \in \mathfrak{F}} \operatorname{Fix}_{A}(f) \neq \emptyset$. Similarly, $\bigcap_{f \in \mathfrak{F}} \operatorname{Fix}_{B}(f) \neq \emptyset$.

Theorem 3.9 Let $X$ be a strictly convex Banach space. Let A, B be nonempty, compact, and convex subsets of $X$ and let $\mathfrak{F}$ be a family of commuting affine and relatively $u$-continuous mappings on $A \cup B$ such that $f(A) \subseteq A$ and $f(B) \subseteq B$. Then there exist points $x_{0} \in A$ and $y_{0} \in B$ such that $x_{0}=f\left(x_{0}\right)$ and $y_{0}=f\left(y_{0}\right)$, for all $f \in \mathfrak{F}$ where $\left\|x_{0}-y_{0}\right\|=\operatorname{dist}(A, B)$.

Proof By Lemma 3.8 the mappings in the family $\mathfrak{F}$ have a common fixed point $x_{0} \in A$, that is, $f\left(x_{0}\right)=x_{0}$ for $f \in \mathfrak{F}$. Let $y_{0} \in B$ be the unique closest point to $x_{0}$ in $B$. Then, for any $f \in \mathfrak{F}$, $\left\|f\left(x_{0}\right)-y_{0}\right\|=\operatorname{dist}(A, B)$, but by the relative $u$-continuity of $f,\left\|f\left(x_{0}\right)-f\left(y_{0}\right)\right\|=\operatorname{dist}(A, B)$. By the uniqueness of the closest point, $y_{0}=f\left(y_{0}\right)$ for $f \in \mathfrak{F}$.

Theorem 3.10 Let $A, B$ be nonempty, compact, and convex subsets of a strictly convex Banach space $X$ and let $\mathfrak{F}$ be a family of commuting, affine and relatively $u$-continuous mappings on $A \cup B$ with $f(A) \subseteq A, f(B) \subseteq B$ for each $f \in \mathfrak{F}$. Let $T: A \rightarrow \mathrm{KC}(B)$ be an upper semicontinuous mapping such that $T(x) \cap B_{0} \neq \emptyset$ for each $x \in A_{0}$. If $\mathfrak{F}$ and $T$ commute, then there exists a point $a \in A$ such that $f(a)=a$ for each $f \in \mathfrak{F}$ and $\operatorname{dist}(a, T(a))=\operatorname{dist}(A, B)$.

Proof By Lemma 3.8, $\bigcap_{f \in \mathfrak{F}} \operatorname{Fix}_{A}(f)$ and $\bigcap_{f \in \mathfrak{F}} \operatorname{Fix}_{B}(f)$ are nonempty.

As in the proof of Theorem 3.3, $T(x)$ is invariant under each $f \in \mathfrak{F}$, for $x \in \operatorname{Fix}_{A}(f)$. Since $\bigcap_{f \in \mathfrak{F}} \operatorname{Fix}_{A}(f) \neq \emptyset$, for $x \in \bigcap_{f \in \mathfrak{F}} \operatorname{Fix}_{A}(f), T(x)$ is invariant under $\mathfrak{F}$. Also, $B_{0}$ is invariant under $\mathfrak{F}$. Therefore as in the proof of Theorem 3.3, since $T(x) \cap B_{0}$ is a compact convex set, $T(x) \cap\left(\bigcap_{f \in \mathfrak{F}} \operatorname{Fix}_{B}(f)\right) \neq \emptyset$. By the proof of Theorem 3.3, $\operatorname{Fix}_{A}(f)$ and $\operatorname{Fix}_{B}(f)$ are compact and convex sets for $f \in \mathfrak{F}$. Therefore, $\bigcap_{f \in \mathfrak{F}} \operatorname{Fix}_{A}(f)$ and $\bigcap_{f \in \mathfrak{F}} \operatorname{Fix}_{B}(f)$ are compact and convex.

Now define $F: \bigcap_{f \in \mathfrak{F}} \operatorname{Fix}_{A}(f) \rightarrow 2^{\bigcap_{f \in \mathfrak{F}} \operatorname{Fix}_{B}(f)}$ by $F(x)=T(x) \cap\left(\bigcap_{f \in \mathfrak{F}} \operatorname{Fix}_{B}(f)\right)$ for each $x \in$ $\bigcap_{f \in \mathfrak{F}} \operatorname{Fix}_{A}(f)$. Clearly, $F$ is an upper semicontinuous multivalued mapping with compact convex values. Now, $P_{A}: \bigcap_{f \in \mathfrak{F}} \operatorname{Fix}_{B}(f) \rightarrow \bigcap_{f \in \mathfrak{F}} \operatorname{Fix}_{A}(f)$. To see this, let $x \in \bigcap_{f \in \mathfrak{F}} \operatorname{Fix}_{B}(f)$. Then $x \in B_{0}$ and $f(x)=x$ for each $f \in \mathfrak{F}$. So, there exists $y \in A$ such that $\|x-y\|=\operatorname{dist}(A, B)$. This implies $x=P_{B}(y)$ and $y=P_{A}(x)$. For each $\delta>0$, we have $\|x-y\|<\delta+\operatorname{dist}(A, B)$. Using the relative $u$-continuity for any $f \in \mathfrak{F}$, $\operatorname{dist}(A, B) \leq\|f(x)-f(y)\|<\epsilon+\operatorname{dist}(A, B)$ for each $\epsilon>0$. Thus, $\|f(x)-f(y)\|=\operatorname{dist}(A, B)$. Therefore, $f(y)=P_{A}(f(x))$ and $f(x)=P_{B}(f(y))$ for each $f \in \mathfrak{F}$. Now, $y=P_{A}(x) \Longrightarrow f(y)=f\left(P_{A}(x)\right) \Longrightarrow P_{A}(x)=f\left(P_{A}(x)\right)$ for each $f \in \mathfrak{F}$. Hence, $P_{A}(x) \in \bigcap_{f \in \mathfrak{F}} \operatorname{Fix}_{A}(f)$ for each $x \in \bigcap_{f \in \mathfrak{F}} \operatorname{Fix}_{B}(f)$. Note that $P_{A} \circ F: \bigcap_{f \in \mathfrak{F}} \operatorname{Fix}_{A}(f) \rightarrow$ $2^{\bigcap \in \mathfrak{F} \operatorname{Fix}_{A}(f)}$. By Lemma 2.7, $P_{A} \circ F$ has a fixed point. So, there exists $a \in \bigcap_{f \in \mathfrak{F}} \operatorname{Fix}_{A}(f)$ such that $a \in\left(P_{A} \circ F\right)(a)$. So, $f(a)=a$ for each $f \in \mathfrak{F}$ and $a \in P_{A}(F(a))$, i.e., there exists $b \in F(a)$ such that $a=P_{A}(b)$. Since $b \in F(a), b \in T(a) \cap\left(\bigcap_{f \in \mathfrak{F}} F \operatorname{Fix}_{B}(f)\right)$. So, $b \in T(a), b \in B_{0}$, and $f(b)=b$ for each $f \in \mathfrak{F} . a=P_{A}(b)$ implies $\|a-b\|=\operatorname{dist}(b, A)$. Since $b \in B_{0}$, there exists $a^{\prime} \in A$ such that $\left\|a^{\prime}-b\right\|=\operatorname{dist}(A, B)$. Since $a \in A$ and $T(a) \subseteq B$, we have

$$
\begin{aligned}
\operatorname{dist}(A, B) & \leq \operatorname{dist}(a, T(a)) \\
& \leq\|a-b\| \\
& =\operatorname{dist}(b, A)
\end{aligned}
$$




$$
\begin{aligned}
& \leq\left\|b-a^{\prime}\right\| \\
& =\operatorname{dist}(A, B) .
\end{aligned}
$$

Thus, $\operatorname{dist}(a, T(a))=\operatorname{dist}(A, B)$.

Corollary 3.11 Let A be a nonempty, compact, and convex subset of a strictly convex Banach space $X$ and let $\mathfrak{F}$ be a family of commuting, affine and continuous self-mappings of $A$. Let $T: A \rightarrow \mathrm{KC}(A)$ be an upper semicontinuous mapping. If $\mathfrak{F}$ and $T$ commute, then there exists a point $a \in A$ such that $a=f(a) \in T(a)$ for each $f \in \mathfrak{F}$.

\section{Examples}

Examples 4.1 to 4.4 are related to Theorem 3.3. On other hand, the last two examples are related to Theorem 3.7 (and Theorem 3.10).

Example 4.1 Let $X=\mathbb{R}^{2}$ with the usual metric. The sets $A=\{(x, y): 0 \leq x \leq 4,1 \leq y \leq$ $5\}, B=\{(x, 0): 0 \leq x \leq 4\}$ are nonempty, compact, and convex with $\operatorname{dist}(A, B)=1$. Define $f: A \cup B \rightarrow A \cup B$ by $f(x, y)=\left(\frac{2 x+1}{3}, y\right)$ and $T: A \rightarrow \mathrm{KC}(B)$ by $T(x, y)=[x, 4] \times\{0\}$. Then $T$ is upper semicontinuous and $f$ is relatively $u$-continuous and affine with $f(A) \subseteq A$ and $f(B) \subseteq B$. As $\operatorname{Fix}(f)=\{(1, y): 1 \leq y \leq 5$ or $y=0\}$, we get $x_{0}=(1,1) \in \operatorname{Fix}(f) \cap A, y_{0}=(1,0) \in$ Fix $(f) \cap B$ with $\left\|x_{0}-y_{0}\right\|=1$. In addition, $f$ and $T$ commute. Indeed, $f(T(x, y))=f([x, 4] \times$ $\{0\})=\left\{\frac{2 z+1}{3}: z \in[x, 4]\right\} \times\{0\}$ and $T(f(x, y))=T\left(\frac{2 x+1}{3}, y\right)=\left[\frac{2 x+1}{3}, 4\right] \times\{0\}$. For $z \in[x, 4], \frac{2 z+1}{3} \in$ $\left[\frac{2 x+1}{3}, 3\right] \subseteq\left[\frac{2 x+1}{3}, 4\right]$. Thus, $f(T(x, y)) \subseteq T(f(x, y))$ for each $(x, y) \in A$. Also, $T(x) \cap B_{0} \neq \emptyset$ for each $x \in A_{0}$ since $A_{0}=\{(x, 1): 0 \leq x \leq 4\}$ and $B_{0}=B$. For $(1,1) \in A$, we have $f(a)=a$ and $\operatorname{dist}(a, T(a))=\operatorname{dist}(A, B)=1$.

Example 4.2 Let $X=\mathbb{R}^{2}$ with the usual metric. The sets $A=\{(0, a): 1 \leq a \leq 3\}, B=\{(x, y)$ : $1 \leq x \leq 5,1 \leq y \leq 5\}$ are nonempty, compact, and convex with $\operatorname{dist}(A, B)=1$. Define $f$ : $A \cup B \rightarrow A \cup B$ by $f(x, y)=\left(x, \frac{y+3}{2}\right)$ and $T: A \rightarrow \mathrm{KC}(B)$ by $T(0, a)=[1, a] \times\{3\}$. Then $T$ is upper semicontinuous and $f$ is relatively $u$-continuous and affine with $f(A) \subseteq A$ and $f(B) \subseteq B$. As $\operatorname{Fix}(f)=\{(x, 3): x=0$ or $1 \leq x \leq 5\}$, we get $x_{0}=(0,3) \in \operatorname{Fix}(f) \cap A, y_{0}=(1,3) \in$ Fix $(f) \cap B$ with $\left\|x_{0}-y_{0}\right\|=1$. In addition, $f$ and $T$ commute. Indeed, $f(T(0, a))=f([1, a] \times$ $\{3\})=[1, a] \times\{3\}$ and $T(f(0, a))=T\left(0, \frac{a+3}{2}\right)=\left[1, \frac{a+3}{2}\right] \times\{3\}$. For $a \in[1,3], \frac{a+3}{2} \geq a$, i.e., $[1, a] \subseteq\left[1, \frac{a+3}{2}\right]$. Thus, $f(T(0, a)) \subseteq T(f(0, a))$ for each $(0, a) \in A$. Also, $T(x) \cap B_{0} \neq \emptyset$ for each $x \in A_{0}$ since $A_{0}=A$ and $B_{0}=\{(1, y): 1 \leq y \leq 3\}$. For $a=(0,3) \in A$, we have $f(a)=a$ and $\operatorname{dist}(a, T(a))=\operatorname{dist}(A, B)=1$.

Example 4.3 Let $X=\mathbb{R}^{2}$ with the usual metric. The sets $A=\{(x, y):-1 \leq x \leq-0.04,-5 \leq$ $y \leq 5\}, B=\{(x, y): 0 \leq x \leq 5,-5 \leq y \leq 5\}$ are nonempty, compact, and convex with $\operatorname{dist}(A, B)=0.04$. Define $f: A \cup B \rightarrow A \cup B$ by $f(x, y)=\left(x, \frac{y+1}{2}\right)$ and $T: A \rightarrow \mathrm{KC}(B)$ by $T(x, y)=\left[0, x^{2}\right] \times\{y\}$. Then $T$ is upper semicontinuous and $f$ is relatively $u$-continuous and affine with $f(A) \subseteq A$ and $f(B) \subseteq B$. As $\operatorname{Fix}(f)=\{(x, 1):-1 \leq x \leq-0.04$ or $0 \leq x \leq 5\}$, we get $x_{0}=(-0.04,1) \in \operatorname{Fix}(f) \cap A, y_{0}=(0,1) \in \operatorname{Fix}(f) \cap B$ with $\left\|x_{0}-y_{0}\right\|=0.04$. In addition, $f$ and $T$ commute. Also, $T(x) \cap B_{0} \neq \emptyset$ for each $x \in A_{0}$ since $A_{0}=\{(-0.04, y):-5 \leq y \leq 5\}$ and $B_{0}=\{(0, y):-5 \leq y \leq 5\}$. For $a=(-0.04,1) \in A$, we have $f(a)=a$ and $\operatorname{dist}(a, T(a))=$ $\operatorname{dist}(A, B)=0.04$. 
Example 4.4 Let $X=\mathbb{R}^{2}$ with the usual metric. The sets $A=\{(x, y):-3 \leq x \leq 3,-1 \leq$ $y \leq-0.25\}, B=\{(x, y):-3 \leq x \leq 3,0 \leq y \leq 4\}$ are nonempty, compact, and convex with $\operatorname{dist}(A, B)=0.25$. Define $f: A \cup B \rightarrow A \cup B$ by $f(x, y)=\left(\frac{x}{2}, y\right)$ and $T: A \rightarrow \operatorname{KC}(B)$ by $T(x, y)=$ $\{x\} \times\left[0, y^{2}\right]$. Then $T$ is upper semicontinuous and $f$ is relatively $u$-continuous and affine with $f(A) \subseteq A$ and $f(B) \subseteq B$. As $\operatorname{Fix}(f)=\{(0, y): 0 \leq y \leq 4$ or $-1 \leq y \leq-0.25\}$, we get $x_{0}=(0,-0.25) \in \operatorname{Fix}(f) \cap A, y_{0}=(0,0) \in \operatorname{Fix}(f) \cap B$ with $\left\|x_{0}-y_{0}\right\|=0.25$. In addition, $f$ and $T$ commute. Also, $T(x) \cap B_{0} \neq \emptyset$ for each $x \in A_{0}$ since $A_{0}=\{(x,-0.25):-3 \leq x \leq 3\}$ and $B_{0}=\{(x, 0):-3 \leq x \leq 3\}$. For $a=(0,-0.25)$, we have $f(a)=a$ and $\operatorname{dist}(a, T(a))=\operatorname{dist}(A, B)=$ 0.25 .

Example 4.5 Let $X=\mathbb{R}^{2}$ with the usual metric. The sets $A=\{(x, y): 0 \leq x \leq 5, y=-1\}$, $B=\{(x, y):-5 \leq x \leq 0, y=1\}$ are nonempty, compact, and convex subsets of a strictly convex Banach space with $\operatorname{dist}(A, B)=2$. Define $f, g: A \cup B \rightarrow A \cup B$ by $f(x, y)=\left(\frac{2 x}{5}, y\right)$ and $g(x, y)=\left(\frac{x}{2}, y\right)$. Then $f, g$ are relatively $u$-continuous and affine with $f(A) \subseteq A, f(B) \subseteq$ $B, g(A) \subseteq A$, and $g(B) \subseteq B$. Also $f, g$ commute. Now, define $T: A \rightarrow \mathrm{KC}(B)$ by $T(x, y)=$ $[-5,-x] \times\left\{y^{2}\right\}$. Then $T$ is upper semicontinuous with nonempty, compact, and convex values. In addition, $T$ commutes with $f$ and $g$. Clearly, $A_{0}=\{(0,-1)\}, B_{0}=\{(0,1)\}$, and $(0,1) \in T(0,-1)=[-5,0] \times\{1\}$. For $a=(0,-1) \in A$ and $b=(0,1) \in B$, we have $f(a)=g(a)=$ $a, f(b)=g(b)=b$, and $\|a-b\|=\operatorname{dist}(A, B)=2$. Moreover, $\operatorname{dist}(a, T(a))=\operatorname{dist}(A, B)$.

Example 4.6 Let $X=\mathbb{R}^{2}$ with the usual metric. The sets $A=\{(x, y):-4 \leq x \leq-1,-6 \leq$ $y \leq 6\}, B=\{(x, y): 0 \leq x \leq 4,-6 \leq y \leq 6\}$ are nonempty, compact, and convex subsets of a strictly convex Banach space with $\operatorname{dist}(A, B)=1$. Define $f, g: A \cup B \rightarrow A \cup B$ by $f(x, y)=$ $\left(x, \frac{y}{3}\right)$ and $g(x, y)=\left(x, \frac{y}{2}\right)$. Then $f, g$ are relatively $u$-continuous and affine with $f(A) \subseteq A$, $f(B) \subseteq B, g(A) \subseteq A$, and $g(B) \subseteq B$. Also $f, g$ commute. Now, define $T: A \rightarrow \mathrm{KC}(B)$ by $T(x, y)=[0,-x] \times\{y\}$. Then $T$ is upper semicontinuous with nonempty, compact, and convex values. In addition, $T$ commutes with $f$ and $g$. Clearly, $A_{0}=\{(-1, y):-6 \leq y \leq 6\}$, $B_{0}=\{(0, y):-6 \leq y \leq 6\}$. So, $(0, y) \in T(-1, y) \cap B_{0}=([0,1] \times\{y\}) \cap B_{0}$ for each $(-1, y) \in A_{0}$. For $a=(-1,0) \in A$ and $b=(0,0) \in B$, we have $f(a)=g(a)=a, f(b)=g(b)=b$, and $\|a-b\|=$ $\operatorname{dist}(A, B)=1$. Moreover, $\operatorname{dist}(a, T(a))=\operatorname{dist}(A, B)$.

\section{Competing interests}

The authors declare that they have no competing interests.

\section{Authors' contributions}

All authors contributed equally and significantly in writing this article. All authors read and approved the final manuscript.

\section{Author details}

'Department of Mathematics, Faculty of Science, King Abdulaziz University, P.O. Box 80203, Jeddah, 21589, Saudi Arabia. 2 Department of Mathematics, Princess Nourah bint Abdulrahman University, P.O. Box 105862, Riyadh, 11656, Saudi Arabia ${ }^{3} 528$ Rover Boulevard, Los Alamos, NM 87544, USA. ${ }^{4}$ Operator Theory and Applications Research Group, Department of Mathematics, Faculty of Science, King Abdulaziz University, P.O. Box 80203, Jeddah, 21589, Saudi Arabia.

\section{Acknowledgements}

This article was funded by the Deanship of Scientific Research (DSR), King Abdulaziz University, Jeddah. The authors, therefore, acknowledge with thanks DSR for technical and financial support. The authors are grateful to three anonymous referees for their useful suggestions and comments.

Received: 29 June 2015 Accepted: 11 December 2015 Published online: 12 January 2016

\section{References}

1. Abkar, A, Gabeleh, M: Global optimal solutions of noncyclic mappings in metric spaces. J. Optim. Theory Appl. 153, 298-305 (2012)

2. Abkar, A, Gabeleh, M: The existence of best proximity points for multivalued non-self-mappings. Rev. R. Acad. Cienc. Exactas Fís. Nat., Ser. A Mat. 107, 319-325 (2013) 
3. Al-Thagafi, MA, Shahzad, N: Best proximity pairs and equilibrium pairs for Kakutani multimaps. Nonlinear Anal. 70, 1209-1216 (2009)

4. Amini-Harandi, A: Best proximity pair and coincidence point theorems for nonexpansive set-valued maps in Hilbert spaces. Bull. Iran. Math. Soc. 37, 229-234 (2011)

5. De la Sen, M: Some results on fixed and best proximity points of multivalued cyclic self-mappings with a partial order. Abstr. Appl. Anal. 2013, Article ID 968492 (2013)

6. Kirk, WA, Reich, S, Veeramani, P: Proximinal retracts and best proximity pair theorem. Numer. Funct. Anal. Optim. 24(7-8), 851-862 (2003)

7. Włodarczyk, K, Plebaniak, R, Obczyński, C: Convergence theorems, best approximation and best proximity for set-valued dynamic systems of relatively quasi-asymptotic contractions in cone uniform spaces. Nonlinear Anal. 72 , 794-805 (2010)

8. Eldred, AA, Kirk, WA, Veeramani, P: Proximal normal structure and relatively nonexpansive mappings. Stud. Math. 171, 283-293 (2005)

9. Elderd, AA, Raj, VS, Veeramani, P: On best proximity pair theorems for relatively u-continuous mapping. Nonlinear Anal. 74, 3870-3875 (2011)

10. Markin, J, Shahzad, N: Best proximity points for relatively u-continuous mappings in Banach and hyperconvex spaces. Abstr. Appl. Anal. 2013, Article ID 680186 (2013)

11. Al-Thagafi, MA, Shahzad, N: Convergence and existence results for best proximity points. Nonlinear Anal. 70 , 3665-3671 (2009)

12. Al-Thagafi, MA, Shahzad, N: Best proximity sets and equilibrium pairs for a finite family of multimaps. Fixed Point Theory Appl. 2008, Article ID 457069 (2008)

13. Jleli, M, Samet, B: Best proximity points for $\alpha$ - $\psi$-proximal contractive type mappings and applications. Bull. Sci. Math $137,977-995(2013)$

14. De la Sen, M, Karapınar, E: Some results on best proximity points of cyclic contractions in probabilistic metric spaces. J. Funct. Spaces 2015, Article ID 470574 (2015)

15. Derafshpour, M, Rezapour, S, Shahzad, N: Best proximity points of cyclic $\phi$-contractions in ordered metric spaces, Topol. Methods Nonlinear Anal. 37, 193-202 (2011)

16. Karapınar, E: Fixed point theory for cyclic weak $\phi$-contraction. Appl. Math. Lett. 24, 822-825 (2011)

17. Karapınar, E, Erhan, IM: Best proximity point on different type contractions. Appl. Math. Inf. Sci. 5, 558-569 (2011)

18. Raj, VS, Veeramani, P: Best proximity pair theorems for relatively nonexpansive mappings. Appl. Gen. Topol. 10, 21-28 (2009)

19. Lassonde, M: Fixed points for Kakutani factorizable multifunctions. J. Math. Anal. Appl. 152, $46-60$ (1990)

20. Prebieracz, B: A proof of the Mazur-Orlicz theorem via the Markov-Kakutani common fixed point theorem, and vice versa. Fixed Point Theory Appl. 2015, 10 (2015)

\section{Submit your manuscript to a SpringerOpen ${ }^{\circ}$ journal and benefit from:}

- Convenient online submission

Rigorous peer review

- Immediate publication on acceptance

- Open access: articles freely available online

- High visibility within the field

- Retaining the copyright to your article 